\title{
Principles of Bracing in the Early Management of Developmental Dysplasia of the Hip
}

\author{
Rajiv Merchant $^{1}\left(\mathbb{D} \cdot\right.$ Abhinav Singh $^{2} \cdot$ Benan Dala-Ali $^{3} \cdot$ Anish P. Sanghrajka $^{1} \cdot$ Deborah M. Eastwood $^{4}$
}

Received: 14 August 2021 / Accepted: 13 September 2021 / Published online: 11 November 2021

(c) Crown 2021

\begin{abstract}
Bracing is considered a gold standard in treating Developmental Dysplasia of the Hip (DDH) in infants under 6 months of age with reducible hips. A variety of braces are available that work on similar principles of limiting hip adduction and extension. This paper summarises the current evidence regarding bracing in DDH. Most of the literature pertains to the Pavlik harness $(\mathrm{PH})$ and there are few studies for other brace types. Bracing eliminates dislocating forces from the hamstrings, the block to reduction of the psoas and improves the muscle line of pull to stabilise the hip joint. Recent studies have shown no benefit in bracing for stable dysplasia. The rates of PH treatment failure in Ortolani-positive hips have been reported to be high. Barlow positive hips have lower Graf grades and are more amenable to PH treatment. There is consensus that the earlier the diagnosis of DDH and initiation of PH treatment, the better the outcome. Failure rates due to unsuccessful reduction and AVN are higher with treatment initiated after age 4-6 months. Studies have shown no benefits of staged weaning of braces. While there is no maximum time in brace, current consensus suggests a minimum of 6 weeks. The key to successful bracing lies in education and communication with the family.
\end{abstract}

Keywords DDH $\cdot$ Brace $\cdot$ Pavlik Harness $\cdot$ Abduction Brace

\section{Introduction}

Bracing is considered the gold standard in treating DDH in infants less than 6 months of age with reducible hips [1-3]. The infant hip with DDH must be maintained within a safe zone of $100^{\circ}$ flexion and abduction that does not exceed

Rajiv Merchant

merchantrajiv@gmail.com

Abhinav Singh

abhinav.singh@ndorms.ox.ac.uk

Benan Dala-Ali

benan@ doctors.org.uk

Anish P. Sanghrajka

ANISH.SANGHRAJKA@nnuh.nhs.uk

Deborah M. Eastwood

deboraheastwood1@nhs.net

1 Norfolk and Norwich University Hospital, Norwich, UK

2 St. Marys Hospital, Imperial College Healthcare NHS Trust, London, UK

3 Milton Keynes University Hospital, Eaglestone, UK

4 Royal National Orthopaedic Hospital, Stanmore, UK $60^{\circ}[1]$. The use of ultrasonography has improved the detection and grading of acetabular dysplasia with a reduction in delayed diagnosis [4-6]. The use of force or exceeding the safe zone to maintain hip position can lead to complications, such as femoral nerve palsy and avascular necrosis (AVN) [7-10]. It is essential that caregivers are familiar with the different bracing options, their efficacy, indications, use and complications. This paper summarises the current evidence regarding bracing in DDH.

Historically, DDH had been treated by manual reduction of the dislocated hip and rigid positioning in a spica or stirrups [11]. Forceful reductions had a high rate of avascular necrosis [11]. Arnold Pavlik from Czechoslovakia introduced the concept of functional-dynamic reduction and demonstrated a dramatic drop in the incidence of AVN with its use [12]. His seven principles of treatment included 1. Treating the hip with active range of movement, 2. Unforced abduction by flexing the hip and knee with stirrups ensuring reduction, 3 . Gentle abduction and flexion redirects the femoral head into the acetabulum which is held in place by the stirrups 4 . Abduction position determined by the infant, 5 . The device allows easy hygiene, 6 . It is simple and can be applied easily by parents and 7. Inexpensive [12]. 


\section{Types of braces}

Brace selection is influenced by the size of the infant, severity of dysplasia, physician preference, local availability and affordability. It is difficult to compare braces and define successful outcomes due to heterogeneity in patient selection and lack of consensus on treatment indications $[3,13]$. The simplest form of bracing is double diapering which has not been shown to be useful maintaining hip reduction [14]. Figure 1 shows some commonly used braces.

Braces can be divided into dynamic and static. Dynamic braces allow the child's legs to move within the range permitted by the brace whilst restricting adduction and extension. This is favoured in new-borns with typically lower complication rates than static braces $[15,16]$. Static bracing holds the hip in a fixed position using rigid supports. Rigid positioning is advantageous in larger infants close to walking age in whom dynamic bracing is not possible
[3]. It is also beneficial in unstable hips that have failed dynamic braces [17-20].

The mechanism by which a static splint may succeed where the PH failed is unclear. A rigid brace generally holds hips in less flexion than a standard PH and may be useful for inferior dislocations as they 'rigidly' hold the hip position $[17,21]$.

Two recent comprehensive systematic reviews on braces available to treat DDH have used contrasting outcome measures. Pavone et al. [22] defined their primary outcome measure as "regression of dysplasia". Ashoor et al. [13] used the crude failure rate as their primary outcome measure. Both studies provided evidence comparing the efficacy of different braces. Their results should be interpreted with caution as normal measurements on ultrasound in infancy do not always correlate with normal radiographs in later followup [23]. Therefore, the concept of success and failure of brace treatment should not be treated as a binary entity but a continuum of improving dysplastic parameters. Table 1
Fig. 1 Commonly used DDH braces

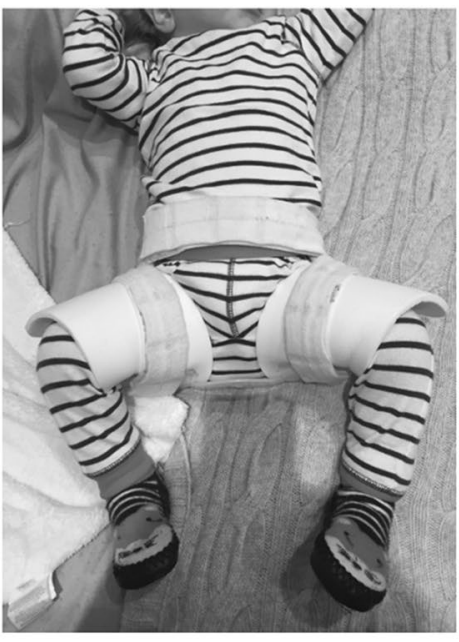

Abduction Brace

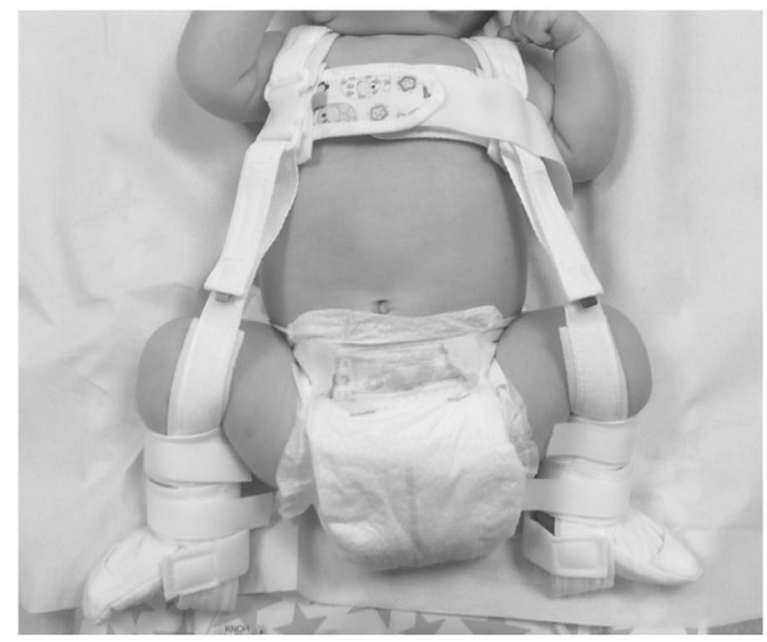

Pavlik Harness

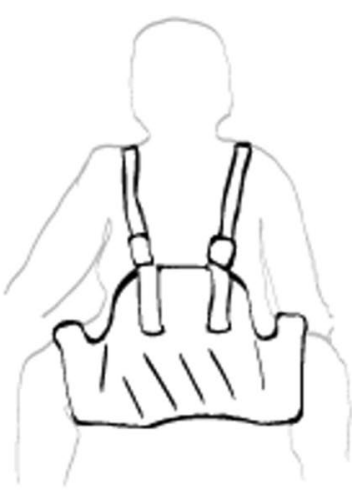

Frejka Pillow

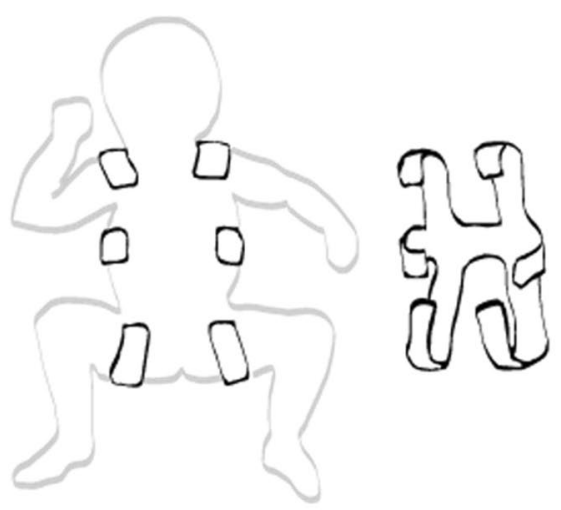

Von Rosen Splint 
Table 1 Success and failure rates of different braces along with documented complications

\begin{tabular}{|c|c|c|c|c|}
\hline Name of Brace & Success rate [22] & Failure rate [13] & Pooled AVN rate [13] & Comments \\
\hline \multicolumn{5}{|l|}{ Dynamic Braces } \\
\hline Pavlik Harness & $91.6 \%$ of 4779 hips & $11.6 \%$ of 10,701 hips & $5.1 \%$ of 10,701 hips & Most used brace \\
\hline Von Rosen Splint & $100 \%$ of 333 patients & $3.5 \%$ of 954 hips & $1.1 \%$ of 639 hips & \\
\hline Tübingen Splint & $97.5 \%$ of 713 hips & $7.79 \%$ of 1001 hips & $0.5 \%$ of 951 hips & \\
\hline Frejka Pillows & $89-97 \%$ of 436 hips & $7.2 \%$ of 606 hips & $1.15 \%$ of 606 hips & \\
\hline Aberdeen Splint & $98.3 \%$ of 120 hips & $4.1 \%$ of 145 hips & $0 \%$ of 145 hips & \\
\hline Coxaflex & $98.3 \%$ of 59 hips & NA & NA & No complications reported [82] \\
\hline Tueffel splint & $100 \%$ of 59 hips & NA & NA & No complications observed [82] \\
\hline \multicolumn{5}{|l|}{ Static Braces } \\
\hline Abduction Brace & $96.8 \%$ of 160 hips & NA & NA & $\begin{array}{l}5 \text { failures including } 2 \text { reported } \\
\text { AVN cases }[17,18]\end{array}$ \\
\hline Rhino Brace & $87.5 \%$ of 40 hips & NA & NA & No complications reported [83] \\
\hline Ifeld splint & $82.1 \%$ of 20 hips & NA & NA & No complications reported (70) \\
\hline
\end{tabular}

describes the pooled outcomes in terms of success and failure represented in the two systematic reviews [3, 13].

\section{Complications of bracing}

AVN can affect the outcome of the hip in adulthood [24]. It is mainly associated with dislocated hips and rarely seen in mild-moderate dysplasia [25]. An adduction contracture (defined as abduction $<60^{\circ}$ when hip flexed to $90^{\circ}$ ) is the most reliable clinical predictor for AVN, likely due to the increased force required to maintain hip abduction [26]. Femoral nerve palsy occurs as a result excessive hip flexion with rates of $2.5 \%$ [1, 27]. In dislocated and irreducible hips treated with $\mathrm{PH}$, Aarvold et al. reported 3/48 cases whilst Novais et al reported 4/78 femoral nerve palsies in patients with dislocated but reducible hips [19].

\section{Biomechanical principles of bracing}

Orlando et al. [28] created a computational model of a pelvis with a dysplastic hip and recreated muscle forces acting for (or preventing) a concentric reduction in PH. They found at $90^{\circ}$ flexion, the iliopsoas was removed as a block to reduction. The pectineus muscle provided the highest component of pull assisting hip reduction. Graf grade III hips were dynamically reducible in $\mathrm{PH}$. The line of pull of all muscles contributed negatively in Graf IV hips explaining why a reduction was less likely to occur in a PH. Hip flexion also eliminates tight hamstrings that may further act as a dislocating force. [14, 22, 29].

Vaefian et al. [30] using finite element analysis simulated contact cartilage pressures (CCP) at different hip flexion and abduction angles. With increasing flexion, the CCP shifted inferiorly leaving the supero-lateral acetabulum unloaded. Assuming that pressure inhibits growth, this CCP distribution would promote deepening of the acetabulum, which is the aim of PH treatment. With increasing abduction, the overall contact area increased in size and CCP. The CCP increased in a non-linear manner to extreme levels in abduction beyond $80^{\circ}$.[31] This increase was in the lateral femoral head where the femoral head blood supply is located.

Kreuz et al. [32] measured axial compressive forces transmitted by harness straps in the Tübingen splint which like the PH relies on shoulder straps to maintain hip abduction and flexion. They found that the shoulders of the new-born infants are loaded with a maximum of $93.9 \%$ of their body mass or $32.3 \mathrm{~N}$ force. In experimental studies, the cadaveric spine buckled under a load greater than $20 \mathrm{~N}$ [33]34. While no adverse impact on the developing spine has been documented, forces transmitted through the shoulders may cause injury to the brachial plexus [35]. The infant must be examined thoroughly at each clinical check and the harness applied with care.

\section{Bracing for stable dysplasia}

In stable dysplasia, the acetabulum demonstrates dysplasia on ultrasound without dislocation, typically defined as Graf II (Fig. 2); a Morin's femoral head-to-acetabular diameter ratio also called Femoral Head Coverage (FHC) index of $\leq 40 \%$ (Fig. 3) or $<2 \mathrm{~mm}$ displacement of the femoral head from the acetabular floor during the Barlow manoeuvre (Fig. 4) [4, 5, 36].

Recent studies have not shown any benefit in bracing for stable dysplasia. [36, 37] Pollet et al. [37] randomised 104 patients aged 3-4 months with stable Graf IIB and IIC hips into active surveillance or PH groups. They found no 


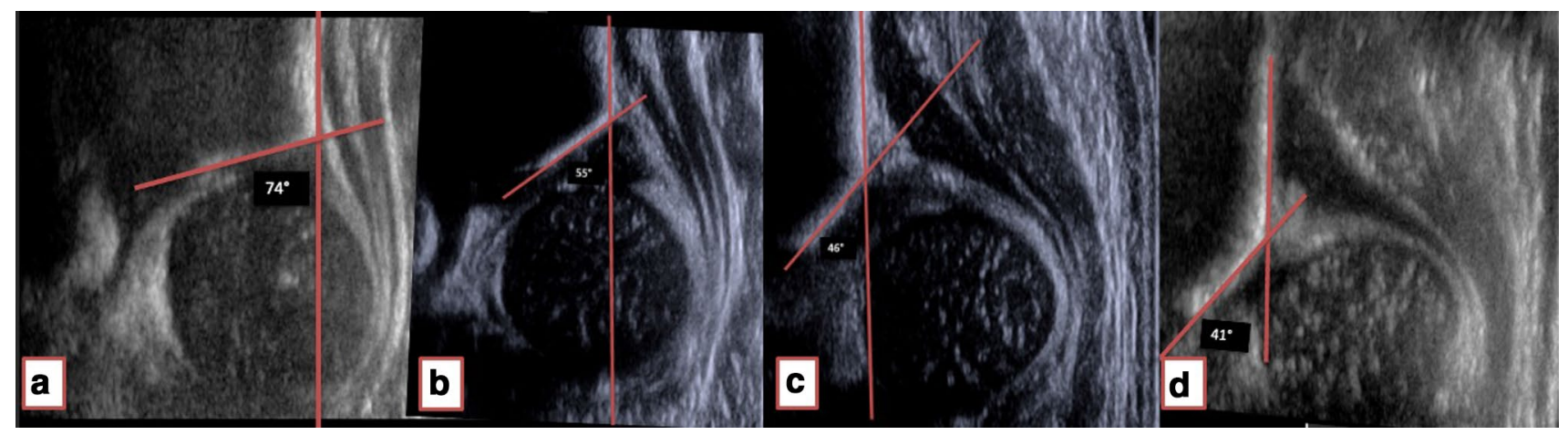

Fig. 2 Representative images of the 4 different Graf Ultrasound grades $(\mathbf{a}=$ Graf I, $\mathbf{b}=$ Graf II, $\mathbf{c}=$ Graf III, $\mathbf{d}=$ Graf IV $)$

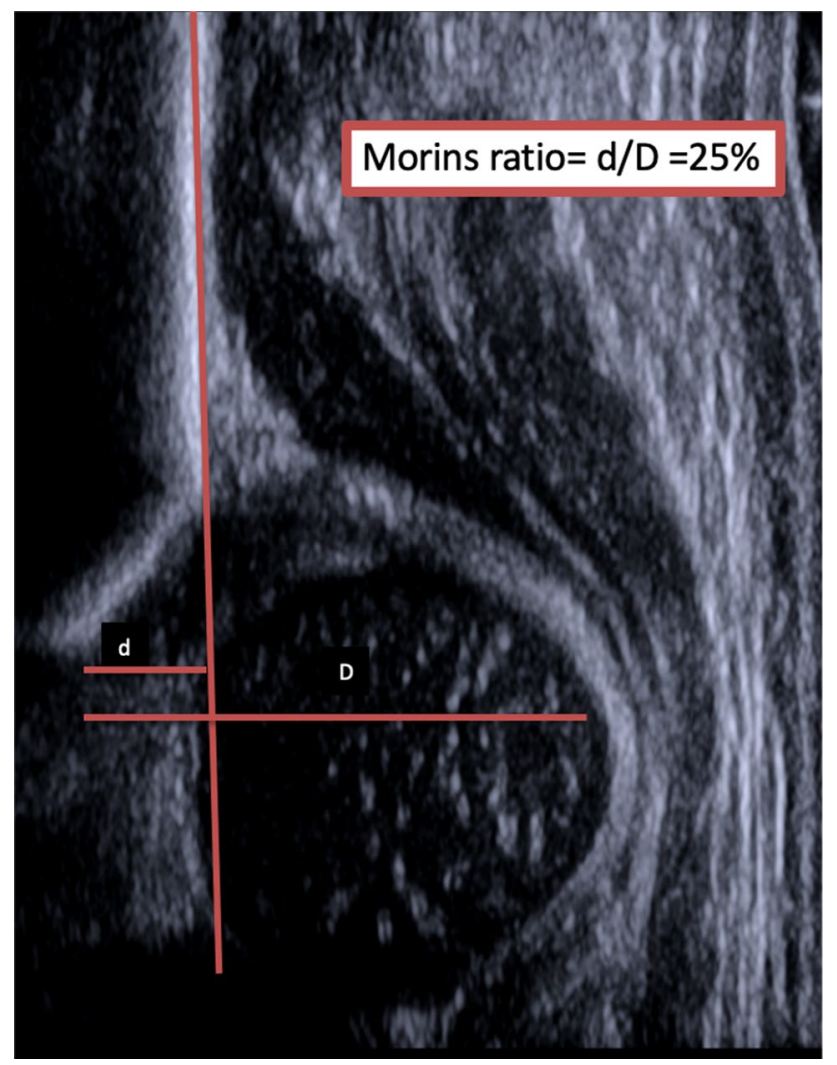

Fig. 3 Representative image of Morin's Ratio/Femoral Head Coverage Index

differences between groups at 6 and 12 week US checks or radiographic parameters at 2 years. Three of 49 patients from active surveillance group were harnessed within the first 6 weeks for deterioration in dysplastic parameters and seven were braced after study period. They recommend active surveillance for patients with stable dysplasia with well-centered hips [37].

Wood et al. [36] compared bracing and observation in infants between 2 and 6 weeks of age, with stable dysplastic hips. They found a statistical difference in Morin's ratio between the two groups, however, in both groups, ratios were above the normal range and not relevant clinically. There was no statistically significant difference in acetabular index at 3- and 24-month follow-up [36].

\section{Bracing for unstable dysplasia (Ortolani and/ or Barlow positive hips)}

The rates of $\mathrm{PH}$ treatment failure in Ortolani-positive hips are reported as high (30-60\%) [19]. This is related to high Graf grades and inability to achieve a stable reduction in harness. The applicability of the Graf classification where the dysplasia is severe (Grade IV) and the hip is reducible (Ortolani-positive) is limited. In this situation, FHC index is more useful. Ortolani-positive patients have an average FHC index between 23 and $37 \%$ with the 90th centile $\leq 33 \%$ FHC [38].

Factors associated with failure for the Ortolani-positive hips include lack of adequate abduction at treatment initiation and complications such as femoral nerve palsy [10,26]. If an Ortolani-positive hip is not reduced and the femoral head subluxated posteriorly in $\mathrm{PH}$, posterior acetabular wall erosion may occur [39]. This may be overcome by using a rigid/static brace, however, flexion is not well controlled in a rigid orthosis and this can lead to AVN [17]40. A systematic review found a significant number of authors reported success in treating dislocated hips with a static brace [41] with $82 \%$ of PH failed hips responding to static bracing. Static bracing should be abandoned early if the hip fails to reduce [18, 41, 42].

Barlow positive hips usually have lower Graf grades and are more amenable to successful PH treatment [1, 19, 29, 42-45].

Dislocated, Ortolani negative (irreducible), hips represent the most severe form of dysplasia. In these patients, $\mathrm{PH}$ can be used safely and successfully in infants treated 


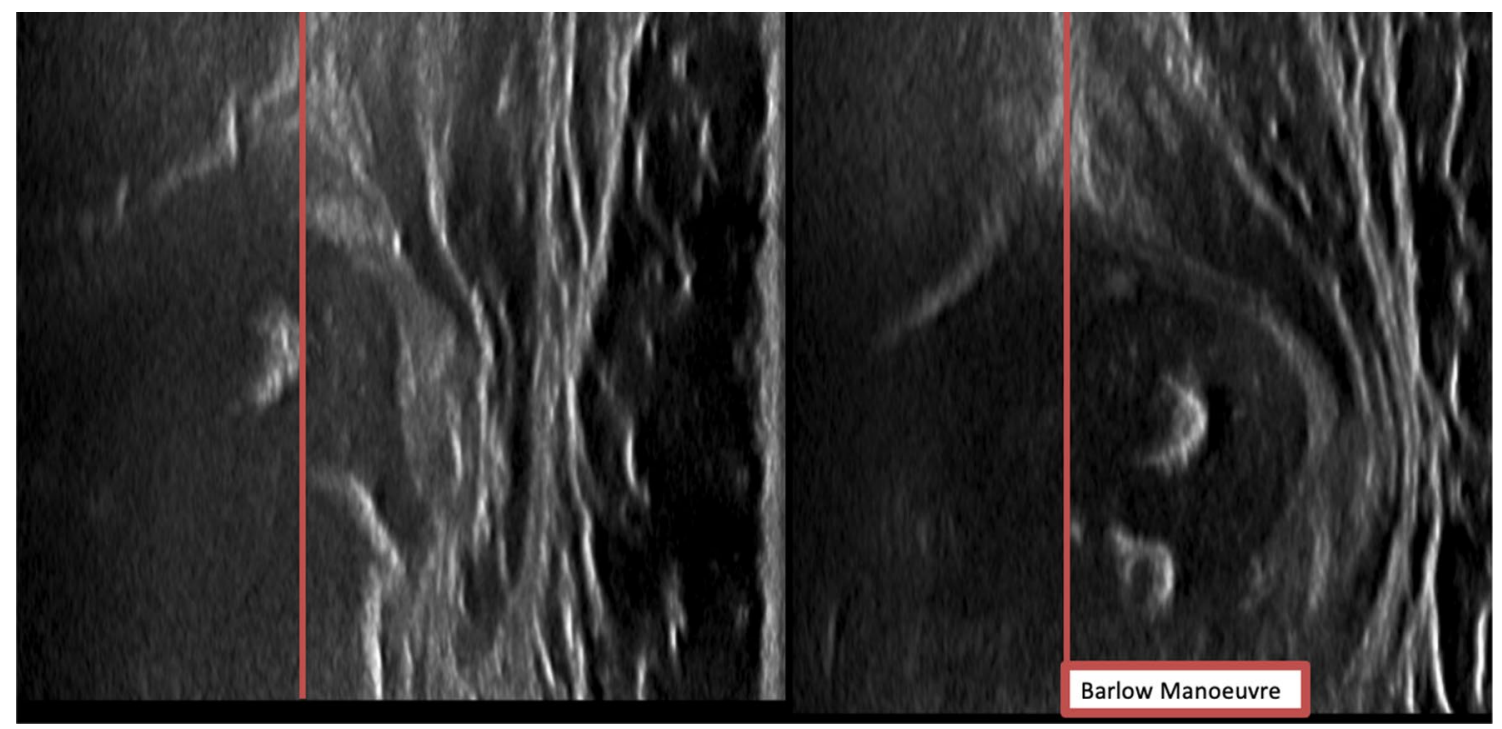

Fig. 4 Ultrasound image showing a Barlow positive hip subluxating

before 4 months of age [25]. Aarvold et al. [25] reported on 48 hips such hips: 27 were treated successfully in $\mathrm{PH}$ and 21 failed $\mathrm{PH}$, of which, 2 had successful static bracing, 7 closed reductions and 12 open reductions [25].

\section{When to start bracing}

The earlier the diagnosis of DDH and initiation of brace treatment, the better the outcomes [4, 6, 46-49]. A late diagnosis is associated with longer, more complex treatment [50]. Failure rates from unsuccessful reduction and AVN are higher with treatment initiated after 4-6 months of age [51-53]. The ideal time for diagnosis and treatment is before 6 weeks of age [54] and the success rate of conservative treatment falls significantly if initiated after seven weeks [21, 55-57]. The more severe the deformity, the earlier the treatment should be started [21, 26, 44, 55].

\section{Duration of brace treatment}

There is considerable variability in the duration of PH/ abduction braces use. Carmichael et al. [58] showed successful resolution of dysplasia at 1 year when bracing was stopped after achieving normality on US. Treatment times averaged 7-8 weeks [58]. While there is no maximum time in brace, the current consensus suggests a minimum period of 6 weeks [59]. However, a considerable number of clinicians brace for a minimum prescribed 12 week period [60]. A systematic review on bracing reported the average fulltime use for dynamic bracing was 16.4 weeks and static bracing was 8.9 weeks [41].
A recent study found introducing US follow-up at 6 weeks significantly reduced the median time in brace from 12 to 6 weeks with no significant increase in dysplastic features at one-year follow-up [61].

\section{Part-time or full-time bracing}

Parental education regarding harness application and care is essential for compliance [62-64]. In children that are tolerant and of suitable size, $\mathrm{PH}$ application is usually full time with one hour in the day for bathing/hygiene purposes. There is no difference between 23 and $24 \mathrm{~h}$ /day harnessing [65]. In children with Ortolani-positive hips, harnesses should not be removed by parents due to the risk of posterior wall erosion and higher failure rates [19].

In older children with residual dysplasia after PH treatment, an abduction brace orthosis may be utilised [66]. This is dependent on the parent and infants ability to cope with bracing as they approach walking age. Studies have shown there is a dose-dependant relationship between improving radiologic parameters and time in harness [67]. However, a pragmatic approach is essential and bracing may be abandoned if a child is intolerant of the orthosis.

\section{Frequency of follow-up in brace}

There is limited evidence on the optimal timing or frequency of follow-up and imaging after commencing PH. Regular reviews provide the opportunity to screen for potential complications and reduce the time in the harness [23, 61, 68]. 
A recent systematic review suggested weekly or 2 weekly examinations with US in harness [27].

A survey of surgeons showed the most common time to review the child after applying a harness was one week and the most common time to repeat the ultrasound was one week in the US and four weeks in Europe [60]. A study looking at the PH efficacy found that sonographic hip stabilization was achieved in $87.4 \%$ of 547 patients, mean age $2.3 \mathrm{~m}$, after 4 weeks [69]. A systematic review suggested that the harness should be discontinued if the hip is not reduced within 4 weeks to avoid possible posterior acetabular wall damage [27].

Static bracing is a viable option for treating unstable hips after PH failure [20, 70] and may avoid the need for more invasive treatment methods under general anaesthesia [71]. Malkawi et al. [69] suggest this switch is generally preferred in the US where they would attempt the static brace after 3 weeks compared to those in Europe who would attempt it after 4 weeks. A recent study demonstrated poor results with static splinting after a $\mathrm{PH}$ and felt it was an unnecessary delay to a closed reduction. However, it should be noted that all three of the patients that failed the static bracing had irreducible hips [20].

At our institution, we stratify US examination follow-up depending on the severity of hip dysplasia (Table 2). Hips with stable dysplasia are brought back for US at 6 weeks when most patients come out of harness. This is in agreement with current evidence [61]. Unstable hips are brought back for weekly US and referred to the paediatric orthopaedic clinic if they are not centred by 2 weeks where a decision is made on further management. During the COVID-19 pandemic, we have successfully trialled and implemented video consultations for patients that only need clinical assessment and harness adjustment. This has now been incorporated into our protocol (Table 2).

\section{Weaning of the brace/harness}

The concept of weaning arises from trying to mitigate the potential risk of late residual acetabular dysplasia after normal ultrasound and completion of harness treatment $[72,73]$. The reported rate of late dysplasia is $3-17 \%$ [74-76]. As the acetabulum develops, imaging necessarily changes from ultrasound to radiographs and with ongoing follow-up the clinically relevant radiographic parameters change from acetabular index to centre edge angle [75, 77]. Continued follow-up is necessary and most patients that may need further treatment are identified by 18 months of age [75]. Radiographic dysplasia may be visible up to 5 years of age in $3.7 \%$ of patients $[75,76]$. Studies have shown no benefit at one year between staged weaning versus no weaning $[72,73]$. A consensus report based only on anecdotal evidence, recommended weaning a $\mathrm{PH}$ after normal US [59]. It is not our departmental practice to wean the PH.

Monitoring following brace removal is recommended until normal development is assured but the literature is limited in defining the optimal frequency or duration of followup. Cashman et al. [23] found that most patients with severe late dysplasia $(\mathrm{CEA}<20)$ could be identified by the trend of the AI measured before the age of 18 months and all by CEA measurement at five years. Thus, they suggested radiographic surveillance until five years of age constituted a safe and effective follow-up [23]. The Stanmore protocol identifies the stages in the progression of DDH when imaging could detect treatment failures or complications [78]. They propose that surveillance after successful $\mathrm{PH}$ treatment can be limited to 6 months, 1, 2 and 5 years of age (Fig. 5) [78].

\section{The neonatal DDH clinic}

The key to the success of the PH lies in the education and communication with the family which can be performed in an outpatient setting [79]. A cause of PH failure is often the parents' low compliance with brace use. Parents play a key role in the effective use of the splint, and they must be educated about its proper use to increase the chance of success [63]. A prospective study found that active maternal participation under direct supervision of an orthopaedic surgeon, could ensure a satisfactory outcome and reported an overall $94 \%$ complete compliance rate [80]. A retrospective study found that Ortolani and Barlow testings were more sensitive when performed by an orthopaedic surgeon compared to other healthcare specialists [34]. At our institution, the neonatal DDH clinic is run by a trained extended scope physiotherapist, nurse practitioners and a radiologist. $\mathrm{We}$ believe that the PH can safely be initiated and managed by an experienced multi-disciplinary team supervised by an orthopaedic surgeon.

\section{Treatment protocols}

The AAOS has the most extensive guidelines on the appropriateness use criteria (AUC) for different treatment modalities for DDH in infants under 6 months [81]. We only identified one other published protocol for $\mathrm{PH} /$ Brace treatment [56] which is broadly similar to our departmental protocol (Table 2).

\section{Summary}

Bracing in early DDH is considered the gold standard treatment method with low complication rates. However, there is a lack of high quality evidence to support this belief due to the heterogeneity of patient populations reported in the 


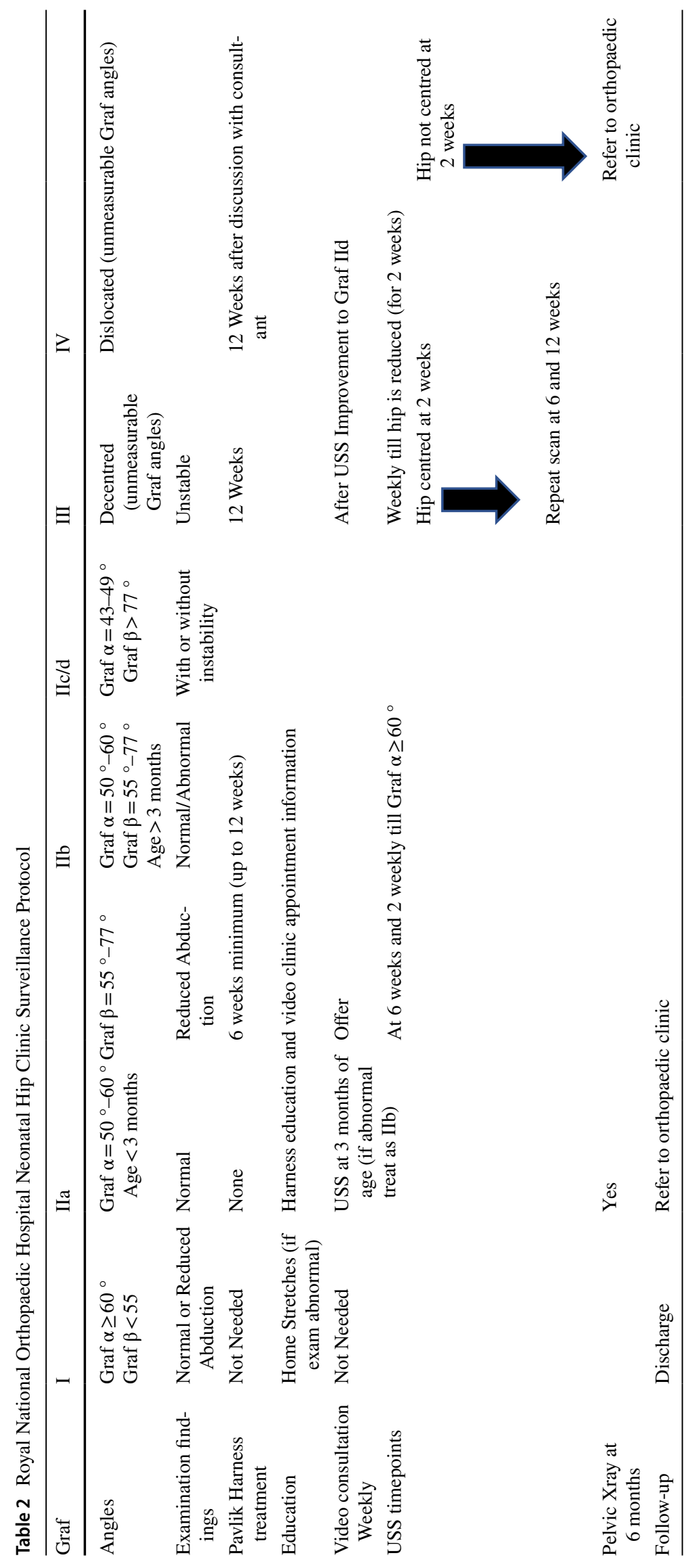




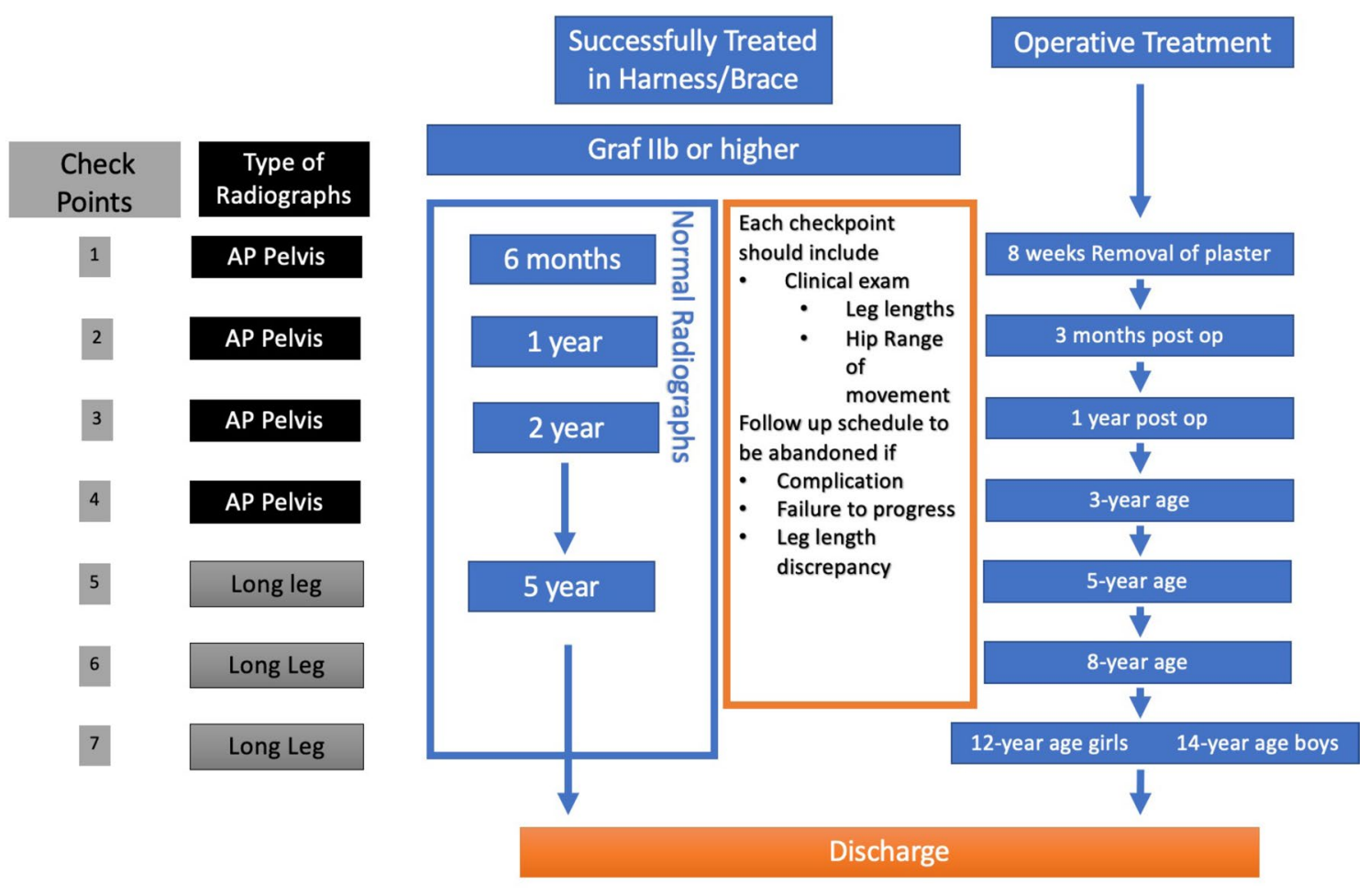

Fig. 5 RNOH Stanmore post brace/harness and operative treatment follow-up schedule

literature and the variations in practice. $\mathrm{PH}$ is the most commonly used brace. There is a wide applicability of $\mathrm{PH}$ in DDH. It should be carried out by experienced caregivers with close follow-up especially in the higher Graf grades. Failure rates are higher with treatment initiated after 4-6 months of age. There is norecommended maximum time for brace use, but the current consensus suggests it should be applied for a minimum of 6 weeks. Education and communication with the family are essential for a successful bracing outcome.

\section{Declarations}

Conflict of interest On behalf of all authors, the corresponding author states that there is no conflict of interest.

Ethical standard statement This article does not contain any studies with human or animal subjects performed by the any of the authors.

Informed consent For this type of study informed consent is not required.

\section{References}

1. Ramsey, P. L., Lasser, S., \& MacEwen, G. D. (1976). Congenital dislocation of the hip. Use of the Pavlik harness in the child during the first six months of life. The Journal of Bone \& Joint Surgery, 58(7), 1000-1004.

2. Sewell, M. D., \& Eastwood, D. M. (2011). Screening and treatment in developmental dysplasia of the hip-where do we go from here? International Orthopaedics, 35(9), 1359-1367.

3. Pavone, V., de Cristo, C., Vescio, A., Lucenti, L., Sapienza, M., Sessa, G., et al. (2021). Dynamic and Static Splinting for Treatment of Developmental Dysplasia of the Hip: A Systematic Review. Children. https://doi.org/10.3390/children8020104

4. Graf, R. (1984). Classification of hip joint dysplasia by means of sonography. Archives of Orthopaedic and Trauma Surgery, $102,248-255$.

5. Graf, R. (2007). Hip sonography: 20 years experience and results. Hip International, 17(SUPPL. 5), 8-14.

6. Eastwood, D. M. (2003). Neonatal hip screening. Lancet, 361(9357), 595-597.

7. Tiruveedhula, M., Reading, I. C., \& Clarke, N. M. P. (2015). Failed Pavlik harness treatment for DDH as a risk factor for avascular necrosis. Journal of Pediatric Orthopedics, 35(2), 140-143.

8. Pool, R. D., Foster, B. K., \& Paterson, D. C. (1986). Avascular necrosis in congenital hip dislocation. The significance of splintage. The Journal of Bone and Joint Surgery British, 68(3), 427-430.

9. Suzuki, R. (1979). Complications of the treatment of congenital dislocation of the hip by the Pavlik harness. International Orthopaedics, 3(1), 77-79.

10. Murnaghan, M. L., Browne, R. H., Sucato, D. J., \& Birch, J. (2011). Femoral nerve palsy in Pavlik harness treatment for developmental dysplasia of the hip. Journal of Bone and Joint Surgery. American Volume, 93(5), 493-499.

11. Mubarak, S. J., \& Bialik, V. (2003). Pavlik: The man and his method. Journal of Pediatric Orthopedics, 23(3), 342-346. 
12. Pavlik, A. (1957). Die funktionelle Behandlungsmethode mittels Riemenbügel als Prinzip der konservativen Therapie bei angeborenen Huftgelenksverrenkungen der Sauglinge. Zeitschrift fur Orthopadie und Ihre Grenzgebiete, 89(3), 341-352.

13. Ashoor, M., Abdulla, N., Elgabaly, E. A., Aldlyami, E., Alshryda, S., Pavone, V., et al. (2020). Evidence based treatment for developmental dysplasia of the hip in children under 6 months of age. Systematic review and exploratory analysis. The Surgeon. https:// doi.org/10.1016/j.surge.2020.02.006

14. De Pellegrin, M., Damia, C. M., Marcucci, L., \& Moharamzadeh, D. (2021). Double Diapering Ineffectiveness in Avoiding Adduction and Extension in Newborns Hips. Children, 8(3), 179.

15. Pagnotta, G., Ruzzini, L., \& Oggiano, L. (2012). Il trattamento dinamico nella displasia evolutiva dell'anca. Archivio di Ortopedia e Reumatologia, 123(1), 21-22. https://doi.org/10.1007/ s10261-012-0010-5

16. Swarup, I., Penny, C. L., \& Dodwell, E. R. (2018). Developmental dysplasia of the hip: An update on diagnosis and management from birth to 6 months. Current Opinion in Pediatrics, 30(1), 84-92.

17. Hedequist, D., Kasser, J., \& Emans, J. (2003). Use of an abduction brace for developmental dysplasia of the hip after failure of Pavlik harness use. Journal of Pediatric Orthopedics, 23(2), 175-177.

18. Eberle, C. F. (2003). Plastazote abduction orthosis in the management of neonatal hip instability. Journal of Pediatric Orthopedics, 23(5), 607-616.

19. Novais, E. N., Kestel, L. A., Carry, P. M., \& Meyers, M. L. (2016). Higher Pavlik Harness Treatment Failure Is Seen in Graf Type IV Ortolani-positive Hips in Males. Clinical Orthopaedics and Related Research, 474(8), 1847-1854.

20. Ibrahim, D. A., Skaggs, D. L., \& Choi, P. D. (2013). Abduction bracing after Pavlik harness failure: An effective alternative to closed reduction and spica casting? Journal of Pediatric Orthopedics, 33(5), 536-539.

21. Viere, R. G., Birch, J. G., Herring, J. A., Roach, J. W., \& Johnston, C. E. (1990). Use of the Pavlik harness in congenital dislocation of the hip. An analysis of failures of treatment. The Journal of Bone and Joint Surgery, 72(2), 238-244.

22. Pavone, V., Testa, G., Riccioli, M., Evola, F. R., Avondo, S., \& Sessa, G. (2015). Treatment of developmental dysplasia of hip with Tubingen hip flexion splint. Journal of Pediatric Orthopedics, 35(5), 485-489.

23. Cashman, J. P., Round, J., Taylor, G., \& Clarke, N. M. P. (2002). The natural history of developmental dysplasia of the hip after early supervised treatment in the Pavlik harness. A prospective, longitudinal follow-up. The Journal of Bone and Joint Surgery. British volume., 84(3), 418-425.

24. Weinstein, S. L., \& Dolan, L. A. (2018). Proximal femoral growth disturbance in developmental dysplasia of the hip: What do we know? Journal of Children's Orthopaedics, 12(4), 331-341.

25. Aarvold, A., Schaeffer, E. K., Kelley, S., Clarke, N. M. P., HerreraSoto, J. A., Price, C. T., et al. (2019). Management of irreducible hip dislocations in infants with developmental dysplasia of the hip diagnosed below 6 months of age. Journal of Pediatric Orthopedics, 39(1), e39-43.

26. Kitoh, H., Kawasumi, M., \& Ishiguro, N. (2009). Predictive factors for unsuccessful treatment of developmental dysplasia of the hip by the Pavlik harness. Journal of Pediatric Orthopedics, 29(6), 552-557.

27. Tibrewal, S., Gulati, V., \& Ramachandran, M. (2013). The Pavlik method: A systematic review of current concepts. Journal of Pediatric Orthopedics. Part B, 22(6), 516-520.

28. Ardila, O. J., Divo, E. A., Moslehy, F. A., Rab, G. T., Kassab, A. J., \& Price, C. T. (2013). Mechanics of hip dysplasia reductions in infants using the Pavlik harness: A physics-based computational model. Journal of Biomechanics, 46(9), 1501-1507. https://doi. org/10.1016/j.jbiomech.2013.03.031

29. Ömeroğlu, H., Köse, N., \& Akceylan, A. (2016). Success of Pavlik Harness Treatment Decreases in Patients $\geq 4$ Months and in Ultrasonographically Dislocated Hips in Developmental Dysplasia of the Hip. Clinical Orthopaedics and Related Research, 474(5), 1146-1152.

30. Vafaeian, B., Adeeb, S., El-Rich, M., Dulai, S. K., \& Jaremko, J. L. (2019). Prediction of mechanical behavior of cartilaginous infant hips in pavlik harness: A subject-specific simulation study on normal and dysplastic hips. J Orthop Res Off Publ Orthop Res Soc., 37(3), 655-664.

31. Vafaeian B, Adeeb S, El-Rich M, Zonoobi D, Hareendranathan AR, Jaremko JL. Hip Joint Contact Pressure Distribution During Pavlik Harness Treatment of an Infant Hip: A Patient-Specific Finite Element Model. J Biomech Eng. 2018 Jul;140(7).

32. Kreuz, P. C., Fröhlich, S., Lindner, T., Olbertz, D., Bader, R., \& Mittelmeier, W. (2012). Biomechanical evaluation of different abduction splints for the treatment of congenital hip dysplasia. Clinical Biomechanics, 27(9), 899-902. https://doi.org/10.1016/j. clinbiomech.2012.06.001

33. Panjabi, M. M., Andersson, G. B., Jorneus, L., Hult, E., \& Mattsson, L. (1986). In vivo measurements of spinal column vibrations. Journal of Bone and Joint Surgery. American Volume, 68(5), 695-702.

34. Orloff, H. A., \& Rapp, C. M. (2004). The effects of load carriage on spinal curvature and posture. Spine, 29(12), 1325-1329.

35. Rizzi, A. M., \& Bielski, R. J. (2020). Brachial Plexus Palsy because of Pavlik harness use: A case report. JBJS case Connector, 10(1), 0579.

36. Wood, M. K., Conboy, V., \& Benson, M. K. D. (2000). Does early treatment by abduction splintage improve the development of dysplastic but stable neonatal hips? Journal of Pediatric Orthopedics, 20(3), 302-305.

37. Pollet, V., Castelein, R. M., van de Sande, M., Witbreuk, M., Mostert, A. K., Besselaar, A., et al. (2020). Abduction treatment in stable hip dysplasia does not alter the acetabular growth: Results of a randomized clinical trial. Science and Reports, 10(1), 9647.

38. Striano, B., Schaeffer, E. K., Matheney, T. H., Upasani, V. V., Price, C. T., Mulpuri, K., et al. (2019). Ultrasound Characteristics of Clinically Dislocated But Reducible Hips With DDH. Journal of Pediatric Orthopedics, 39(9), 453-457.

39. Suzuki, S. (1993). Ultrasound and the Pavlik harness in CDH. Journal of Bone and Joint Surgery. British Volume, 75(3), 483-487.

40. Swaroop, V. T., Mubarak, S. J., Success, I., New, W., \& Protocol, T. (2009). Difficult-to-treat Ortolani-positive hip: Improved success with new treatment protocol. Journal of Pediatric Orthopedics, 29(3), 224-230.

41. Pavone, V., de Cristo, C., Vescio, A., Lucenti, L., Sapienza, M., Sessa, G., et al. (2021). Dynamic and static splinting for treatment of developmental dysplasia of the hip: A systematic review. The Child, 8(2), 104.

42. Grill, F., Bensahel, H., Canadell, J., Dungl, P., Matasovic, T., \& Vizkelety, T. (1988). The Pavlik harness in the treatment of congenital dislocating hip: Report on a multicenter study of the European Paediatric Orthopaedic Society. Journal of Pediatric Orthopedics, 8(1), 1-8.

43. Kalamchi, A., \& Macfarlane, R. (1982). The pavlik harness: Results in patients over three months of age. Journal of Pediatric Orthopedics, 2(1), 3-8.

44. Lerman, J. A., Emans, J. B., Millis, M. B., Share, J., Zurakowski, D., \& Kasser, J. R. (2001). Early failure of Pavlik harness treatment for developmental hip dysplasia: Clinical and ultrasound predictors. Journal of Pediatric Orthopedics, 21(3), 348-353. 
45. Mostert, A. K., Tulp, N. J. A., \& Castelein, R. M. (2002). Early failure of Pavlik harness treatment of developmental hip dysplasia: clinical and ultrasound predictors. Journal of pediatric orthopedics, 22, 410. discusssion 410.

46. Harcke, H. T. (1994). Screening newborns for developmental dysplasia of the hip: The role of sonography. American Journal of Roentgenology, 162(2), 395-397.

47. Gerscovich, E. O. (1997). A radiologist's guide to the imaging in the diagnosis and treatment of developmental dysplasia of the hip. I. General considerations, physical examination as applied to real-time sonography and radiography. Skeletal Radiology, 26(7), 386-397.

48. Rosendahl, K., Aslaksen, A., Lie, R. T., \& Markestad, T. (1995). Reliability of ultrasound in the early diagnosis of developmental dysplasia of the hip. Pediatric Radiology, 25(3), 219-224.

49. Jones, D. (1998). Neonatal detection of developmental dysplasia of the hip (DDH). The Journal of bone and joint surgery. British volume, 80, 943-945.

50. Senaran, H., Bowen, J. R., \& Harcke, H. T. (2007). Avascular necrosis rate in early reduction after failed Pavlik harness treatment of developmental dysplasia of the hip. Journal of Pediatric Orthopedics, 27(2), 192-197.

51. Inoue, T., Naito, M., \& Nomiyama, H. (2001). Treatment of developmental dysplasia of the hip with the Pavlik harness: Factors for predicting unsuccessful reduction. Journal of Pediatric Orthopedics. Part B, 10(3), 186-191.

52. Konigsberg, D. E., Karol, L. A., Colby, S., \& O’Brien, S. (2003). Results of medial open reduction of the hip in infants with developmental dislocation of the hip. Journal of Pediatric Orthopedics, 23(1), 1-9.

53. Kruczynski, J. (1996). Avascular necrosis of the proximal femur in developmental dislocation of the hip. Incidence, risk factors, sequelae and MR imaging for diagnosis and prognosis. Acta Orthopaedica Scandinavica, 268, 1-48.

54. Agostiniani R, Atti G, Bonforte S, Casini C, Cirillo M, De Pellegrin M, et al. Recommendations for early diagnosis of Developmental Dysplasia of the Hip (DDH): working group intersociety consensus document. Ital J Pediatr [Internet]. 2020 Oct 9;46(1):150. Available from: https://pubmed.ncbi.nlm.nih.gov/ 33036652

55. Atalar, H., Sayli, U., Yavuz, O. Y., Uraş, I., \& Dogruel, H. (2007). Indicators of successful use of the Pavlik harness in infants with developmental dysplasia of the hip. International Orthopaedics, $31(2), 145-150$

56. Walton, M. J., Isaacson, Z., McMillan, D., Hawkes, R., \& Atherton, W. G. (2010). The success of management with the Pavlik harness for developmental dysplasia of the hip using a United Kingdom screening programme and ultrasound-guided supervision. Journal of Bone and Joint Surgery, British volume, 92(7), 1013-1016.

57. van der Sluijs, J. A., De Gier, L., Verbeke, J. I., Witbreuk, M. M. E. H., Pruys, J. E. H., \& van Royen, B. J. (2009). Prolonged treatment with the Pavlik harness in infants with developmental dysplasia of the hip. The Journal of Bone and Joint Surgery. British volume, 91(8), 1090-1093.

58. Carmichael, K. D., Longo, A., Yngve, D., Hernandez, J. A., \& Swischuk, L. (2008). The use of ultrasound to determine timing of pavlik harness discontinuation in treatment of developmental dysplasia of the hip. Orthopedics, 31(10), 988.

59. Kelley, S. P., Feeney, M. M., Maddock, C. L., Murnaghan, M. L., \& Bradley, C. S. (2019). Expert-based consensus on the principles of Pavlik harness management of developmental dysplasia of the hip. JBJS Open Access., 4(4), e0054.

60. Alves, C., Truong, W. H., Thompson, M. V., Suryavanshi, J. R., Penny, C. L., Do, H. T., et al. (2018). Diagnostic and treatment preferences for developmental dysplasia of the hip: A survey of
EPOS and POSNA members. Journal of Children's Orthopaedics, 12(3), 236-244.

61. Theunissen, W. W. E. S., Van Der, S. M., Van, D. F. Q. M. P., Witlox, A. M. A., \& Tolk, J. J. (2021). Timing of Repeat Ultrasound Examination in Treatment of Stable Developmental Dysplasia of the Hip. Journal of Pediatric Orthopedics, 41(4), 203-208.

62. McHale, K. A., \& Corbett, D. (1989). Parental noncompliance with Pavlik harness treatment of infantile hip problems. Journal of Pediatric Orthopedics, 9(6), 649-652.

63. Mubarak, S., Garfin, S., Vance, R., McKinnon, B., \& Sutherland, D. (1981). Pitfalls in the use of the Pavlik harness for treatment of congenital dysplasia, subluxation, and dislocation of the hip. Journal of Bone and Joint Surgery. American Volume, 63(8), 1239-1248.

64. Zídka, M., \& Džupa, V. (2019). Pavlik harness and Frejka pillow: Compliance affects results of outpatient treatment. Archives of Orthopaedic and Trauma Surgery, 139(11), 1519-1524.

65. Hines, A. C., Neal, D. C., Beckwith, T., Jo, C. H., \& Kim, H. K. W. W. (2019). A Comparison of Pavlik Harness Treatment Regimens for Dislocated but Reducible (Ortolani+) Hips in Infantile Developmental Dysplasia of the Hip. Journal of Pediatric Orthopedics, 39(10), 505-509.

66. Gans, I., Flynn, J. M., \& Sankar, W. N. (2013). Abduction bracing for residual acetabular dysplasia in infantile DDH. Journal of Pediatric Orthopedics, 33(7), 714-718.

67. Swarup, I., Talwar, D., \& Sankar, W. N. (2021). Part-time abduction bracing in infants with residual acetabular dysplasia: Does compliance monitoring support a dose-dependent relationship? Journal of Pediatric Orthopedics, 41(2), E125-E129.

68. Malkawi, H. (1998). Sonographic monitoring of the treatment of developmental disturbances of the hip by the Pavlik harness. Journal of Pediatric Orthopedics. Part B, 7(2), 144-149.

69. Sankar, W. N., Nduaguba, A., \& Flynn, J. M. (2015). Ilfeld abduction orthosis is an effective second-line treatment after failure of Pavlik harness for infants with developmental dysplasia of the hip. Journal of Bone and Joint Surgery. American Volume, 97(4), 292-297.

70. Olsen, E. A., \& Brambrink, A. M. (2013). Anesthesia for the young child undergoing ambulatory procedures: Current concerns regarding harm to the developing brain. Current Opinion in Anaesthesiology, 26(6), 677-684.

71. Westacott, D. J., Mackay, N. D., Waton, A., Webb, M. S. L., Henman, P., \& Cooke, S. J. (2014). Staged weaning versus immediate cessation of Pavlik harness treatment for developmental dysplasia of the hip. Journal of Pediatric Orthopedics. Part B, 23(2), 103-106.

72. Bram, J. T., Gohel, S., Castañeda, P. G., \& Sankar, W. N. (2021). Is There a Benefit to Weaning Pavlik Harness Treatment in Infantile DDH? Journal of Pediatric Orthopedics, 41(3), 143-148.

73. Tucci, J. J., Kumar, S. J., Guille, J. T., \& Rubbo, E. R. (1991). Late acetabular dysplasia following early successful Pavlik harness treatment of congenital dislocation of the hip. Journal of Pediatric Orthopedics, 11(4), 502-505.

74. Cashman, J. P., Round, J., Taylor, G., \& Clarke, N. M. P. (2002). The natural history of developmental dysplasia of the hip after early supervised treatment in the Pavlik harness. A prospective longitudinal follow-up. The Journal of Bone and Joint Surgery. British volume, 84(3), 418-425.

75. Alexiev, V. A., Harcke, H. T., \& Kumar, S. J. (2006). Residual dysplasia after successful Pavlik harness treatment: Early ultrasound predictors. Journal of Pediatric Orthopedics, 26(1), 16-23.

76. Broughton, N. S., Brougham, D. I., Cole, W. G., \& Menelaus, M. B. (1989). Reliability of radiological measurements in the assessment of the child's hip. Journal of Bone and Joint Surgery. British volume, 71(1), 6-8. 
77. Wright, J., Tudor, F., Luff, T., \& Hashemi-Nejad, A. (2013). Surveillance after treatment of children with developmental dysplasia of the hip: Current UK practice and the proposed Stanmore protocol. Journal of Pediatric Orthopaedics Part B, 22(6), 509-515.

78. Iwasaki, K. (1983). Treatment of congenital dislocation of the hip by the Pavlik harness. Mechanism of reduction and usage. The Journal of Bone \& Joint Surgery., 65(6), 760-767.

79. Hassan, F. A. (2009). Compliance of parents with regard to Pavlik harness treatment in developmental dysplasia of the hip. J Pediatr Orthop Part B, 18(3), 111-115.

80. Samora, J., Quinn, R. H., Murray, J., Pezold, R., \& Hall, Q. (2019). Management of Developmental Dysplasia of the Hip in Infants up to Six Months of Age: Intended for Use by Orthopaedic Specialists. Journal of American Academy of Orthopaedic Surgeons, 27(8), e360-e363.
81. Azzoni, R., \& Cabitza, P. (2011). A comparative study on the effectiveness of two different devices in the management of developmental dysplasia of the hip in infants. Minerva Pediatrica, 63(5), 355-361.

82. Wahlen, R., \& Zambelli, P. Y. (2015). Treatment of the Developmental Dysplasia of the Hip with an Abduction Brace in Children up to 6 Months Old. Advances in Orthopedics, 2015, 103580. https://doi.org/10.1155/2015/103580

Publisher's Note Springer Nature remains neutral with regard to jurisdictional claims in published maps and institutional affiliations. 